\title{
Advances and Developments in the Use of Human Mesenchymal Stem Cells - A Few Considerations
}

\author{
Lucinda Kennard ${ }^{1}$, Gopiga Thanabalasundaram ${ }^{2}$, Hiteshkumar D. Tailor ${ }^{*}, 2$ and Wasim S. Khan ${ }^{3}$ \\ ${ }^{I}$ Foundation Training Department, East of England NHS Deanery, Cambridge, CB21 5XE, UK \\ ${ }^{2}$ University College London Medical School, Gower Street, London WC1E 6BT, UK \\ ${ }^{3}$ University College London Institute of Orthopaedics and Musculoskeletal Sciences, Royal National Orthopaedic \\ Hospital, Stanmore, Middlesex, HA7 4LP, UK
}

\begin{abstract}
One less visited area in musculoskeletal stem cell research is the effects of donor age on quality of stem cells. The prevalence of degenerative orthopaedic conditions is large, and the older population is likely to receive great benefit from stem cell therapies. There are many known growth factors involved in controlling and influencing stem cell growth which are also related to cell senescence. Of which, expressions are found to be altered in mesenchymal stem cells from older donors. Considerations must also be taken of these mechanisms which also have a role in cell cycle and tumour suppression.
\end{abstract}

Keywords: Ageing, bone marrow, donor, growth factors, mesenchymal stem cells, senescence.

Degenerative and many other orthopaedic conditions widely affect the older population. There is a lot of active mesenchymal research in the orthopaedic field looking for ways to combat these diseases. So this review looks at the implications of ageing with regards to mesenchymal stem cells and the impact it has on replication for these cells. In vivo work and the concept of replicative senescence in vitro and techniques researchers are discovering to overcome these problems to help mesenchymal stem cell supply.

\section{EFFECTS OF DONOR STEM CELL AGE ON CELL REPLICATION AND DIFFERENTIATION}

Human Mesenchymal Stem Cells (hMSCs) are clonogenic, proliferate easily and their progenies show a broad multilineage differentiation potential.

\section{In Vitro Studies}

Generally MSCs in the bone marrow are arrested in the G0 state, but when these cells are plated in vitro, MSCs exit their quiescent state and proliferate to form individual colony forming unit-fibroblasts (CFU-Fs). Each of these colonies are derived from a single MSC.

The number of MSCs in the bone marrow has been shown to vary with donor age. But on the other hand there are several studies which also did not detect a difference in the number of MSCs with varying age [1].

D'Ippolito et al. studied the effects of MSCs which were taken from the vertebral bodies of donors [2]. These were then grown in various cultures until they formed colonies which were then plated and promoted to cause osteoblastic differentiation. The cultures were analyzed and the cells with

*Address correspondence to this author at the University College London Medical School, Gower Street, London WC1E 6BT, UK; Tel: +44 207679 0858; Fax: 0207679 0890;

E-mails: mr.htailor@gmail.com, htailor@doctors.org.uk osteogenic potential were looked at. Closer analysis revealed that a higher number of MSCs with osteoblastic potential are present in the younger donor cells in comparison to the older donor cells. This decrease in the potential to differentiate into osteoblasts may partly explain the increased risk of osteoporosis in the older population.

The progenitor cell numbers and the clonogenic potential was compared in donor cells from females under 35 years of age with the donor cells of females at the age of 45 and over [3]. The results showed that the cellular yield is significantly different between the two age groups. Even though both groups had the ability to produce colonies, the younger donor cells demonstrated a doubling in the potential to clone in comparison to the older donor stem cells. In this study specific MSC markers were also analysed using flow cytometry. The following markers were studied: CD13, CD14, CD29, CD34, CD44, CD45, CD49d, CD54, CD71, CD90 and CD105 [4,5]. But none of these showed significant variations related to the donor age of the MSC.

As well as comparing MSC markers, peroxisome proliferator-activated receptor gamma (PPAR- $\gamma$ ) expression in undifferentiated and adipogenic differentiated human adipocytic stem cells were analyzed by De Girolamo et al. [3] and others [6,7]. However, no significant difference in PPAR- $\gamma$ expression of differentiated human stem cells was found between the two age groups in De Girolamo et al's study [3]. This therefore suggests that the proliferation and differentiation potential between both groups is not affected by transcription factors regulating gene expression or by MSC markers.

However, when the human stem cells were cultured in an osteogenic differentiating medium for two weeks, a large difference was detected in the alkaline phosphatase (ALP) activity. The cells from the younger female donors showed an increase of $280 \%$ in ALP activity whereas the older donor 
cells had an increase of $40 \%$ activity only. From this data it is difficult to hypothesize the reasons for the difference seen in the osteoblastic differentiation potential even though the MSC markers did not show any significant difference.

A recent research study by Katsara et al. revealed the presence of Dazi, Oct3/4 and Sox 2 transcription factors in bone marrow-derived MSCs [8]. These are critical for embryonic development. But their quantity was shown to be significantly lower in younger donor stem cells than in older donor stem cells. This therefore may have severe implications on the potential of adult stem cells to be used in regenerative medicine.

We have previously shown that synovial fat pad derived MSCs isolated from two groups of five patients with a mean age of 57 years (SD 3 years) and 86 years (SD 3 years) showed similar proliferation rates and cell surface epitope profile [9]. The cells from both groups cultured in osteogenic medium exhibited osteogenesis as shown by a significant upregulation of alkaline phosphatase and osteocalcin genes, and significantly greater alkaline phosphatase enzyme activity compared to cells cultured in the control medium. The cells cultured in the osteogenic medium also showed greater calcium phosphate deposition on alizarin red staining. There was no significant difference between the osteogenic potential of the two age groups for any of the parameters looked at. We concluded that the synovial fat pad is a source of stem cells that exhibits osteogenic differentiation potential not affected by ageing in later life.

\section{In Vivo Studies}

Even though hMSCs are being studied for several decades, it is only recently that in vivo characteristics of MSCs are considered and outlined. Therefore the number of studies done in vivo is limited. However, several researchers have tried to mimic in vivo properties in order to present the nature of the progenitors as would happen in the human body.

A study by Stolzing et al. looked at the action of hMSCs in vivo [10]. They detected a decrease in stem cell proliferation with increasing age. This is consistent with the finding of an enhancement in the p53 expression and its targets p21 and BAX. These factors are known to have apoptotic actions which explains the results of this study. Similarly, a decrease in the potential to differentiate into osteoblasts was detected.

Osteoprogenitors in the human bone marrow demonstrate the ability to regenerate bone and stroma [11]. These hMSCs express a high number of CD146 adhesion molecules. Following transplantation of CD146 positive stromal cells, they associate with developing sinusoids and eventually regenerate human cells in vivo.

Recent research with hMSC transplantation into cyclosporine-immunosupressed rats after myocardial infarction has shown that hMSCs from donors aged 1-5 years old in comparison to donors aged 50-70 years old, the young hMSCs offer the best preserved ejection fraction, fractional shortening, left ventricular end-diastolic and end systolic tidal volume despite the fact that morphologically the hMSCs were similar regardless of age [12]. This research was based on suggestions from previous studies that donor stem cell age had important impact on the regenerative capacity of stem cell therapies.

Furthermore, it has been estimated that the population doubling of cells from older donors is about twenty five percent lower compared with cells from younger individuals [13]. With ageing, the ability of isolated MSCs to form adherent colonies declines with age. There is a reduction in MSCs numbers as the age of the donor cells increase [14].

\section{The Problem of Senescence in In Vitro Culturing}

The unlimited self-renewal ability of MSCs is a major feature. This gives stem cells the ability to potentially differentiate into any type of cell. They only reach senescence as a cellular response when there is extensive DNA damage which is irreparable. Therefore, it has a role that acts similar to a tumour suppressor. It is an important factor of in vitro studies as it may affect the results due to replicative and stress senescence. It may also have an impact on clinical medicine as MSCs are generally expanded in culture in vitro and then they are introduced in vivo to allow proceeding with its regenerative purpose.

There is a theory which states that the proliferative capacity of hMSCs in vitro is considered to be finite; this is a concept which is known as the "Hayflick limit" [15]. In culture the "Hayflick limit" has been widely cited in papers, stemming from Leonard Hayflick he found that replicative senescence occurs in vitro. In vitro cultured cells proliferate until a limited number of differentiation has occured. Then these hMSCs enter a state which is referred to as 'premature growth arrest'. This can also be termed as senescence - a state where cells are non-responsive to mitogenic stimuli even though they are metabolically active. This is explained by the suggestion that senescence acts a biological protection from neoplastic transformations [16].

Roura et al. studied the effect of ageing on the pluripotential capacity of human CD105+ MSCs as it was unclear whether aging modifies MSCs properties [4]. Cells of young and elderly donors were obtained. The pluripotential capacity was analysed using various induction media. The results indicated no significant differences in telemere length and lipofuscin accumulation. Both, young and older donor-derived cells, showed an increase in the number of connexin-43 without any significant difference. This therefore does not support the presence of senescence in vitro. But on the other hand there are several studies which do provide evidence supporting the "Hayflick limit" theory as outlined below.

Micro-Ribonucleic Acids (miRNAs) are a class of non coding silencing RNAs and a single miRNA can regulate up to several hundred miRNA targets and as such can have a large influence in the cell [17]. It has been shown that miR17, miR-19b, miR-20a and miR-106a are downregulated in hMSCs with age and may contribute to senescence [18].

The expression of senescence associated betagalactosidase is a lysosomal marker of cell senescence in vitro [19]. Forkhead box-M1 (FoxM1) is a transcription factor that is expressed in proliferating cells. This therefore suggests that it may have a role in the regulation of cell division. In fact, its activity starts when a cell enters the $\mathrm{S}$ phase and hence has a vital role in mitosis [19]. Cells in 
which FoxM1 is deleted show signs of difficulty in proliferation and exhibit abnormalities.

Interestingly a study from bone-marrow derived MSCs showed that gene ontology categories relating to DNA repair, mitosis, transcriptional regulation and nucleus were age-repressed in MSCs, whereas genes involved in categories for differentiation, plasma membrane and extra cellular matrix were age induced in MSCs [20]. It has been highlighted that age associated changes differ a lot between cell types; e.g. Haemopoetic Progenitor Cells (HPCs) and MSCs do not have the same age-induced gene expression. Fascinatingly, Hutchinson-Gilford progeria syndrome, a severe autosomal dominant premature ageing condition, is linked to a mutation of LMNA gene. Lamin A and Lamin C are derived from alternative splicing of LMNA. Lamin A/C is crucial for nuclear integrity. In HPCs, lamin A is an age induced gene which is also mutated in Hutchinson-Gilford progeria. In aged induced gene expression of MSCs mesenchyme homeobox (MEOX2) is a negative regulator of proliferation in several mesodermal tissues and is affected in fibroblasts of patients with Hutchison-Gilford Progeria syndrome [21]. Other upregulated genes in MSCs were SHOX2 (short stature homeobox 2) and HOXC6 part of the developmental regulatory system. Age-repressed homeobox genes included HOXA5, HOXB3, HOXB7 and PITX2 (paired-like homeodomain transcription factor 2) [22].

\section{Tactics to Combat Replicative Senescence In Vitro}

It has been shown that hMSCs enhance proliferation capacity when cultured at conditions of low atmospheric oxygen. Human MSCs from cancellous bone cultured in oxygen tension of $3 \%$ showed the rates of cell death and hypoxia induced gene transcription were unchanged, but in vitro proliferation life span was increased with about 10 additional population doublings before reaching terminal growth arrest [23]. Similarly, whole genome expression profiles of primary MSCs from infants and adults have also been compared after a short period of atmospheric conditions of high and low oxygen tensions. Leptin receptor (LEPR) gene expression was increased in MSCs from aged donors in aged cells and in those cultivated at elevated oxygen levels. Dying MSCs expressed enhanced levels of LEPR. This fits in line with low oxygen tension culturing being beneficial. Furthermore, LEPR mRNA levels could be used as a measure of stem cell ageing to help find more tactics to beat stem cell senescence [24].

Cell density in culture has an impact on replicative sensensce. Colter et al. [25] showed that hMSCs propagate much more rapidly if they are plated at low densities of 1.5 or 3.0 cells $/ \mathrm{cm}^{2}$. They showed that single cell derived MSC clones could be expanded up to 50 PDs in about 10 weeks if cultured by repeat passages at low density whereas cells stopped growing after 15 passages at high cell density.

Simonson et al. [26] showed that when hMSCs were transduced by a retroviral vector containing a gene for the catalytic subunit of human telomerase, hTERT, these cells underwent more than 260 population doublings. However, control cells underwent senescence associated proliferation arrest at 26 population doublings. These cells also maintained their osteogenic potential.
Furthermore, a study that introduced Wild type p53 Inducibile Phosphatase 1 (Wip1), showed that, it lowered p16INKa expression and led to p38 MAPK inactivation in hMSCs and successfully delayed cell growth arrest of these cells in prolonged culture. P16INK4a is a known tumour suppressor that inhibits G1 cyclin dependent kinase (CDK) function, leading to the activation of the retinoblastoma protein $(\mathrm{Rb})$, eventually leading to premature growth arrest. Interestingly, Wip1 failed to induce re-entry of post senescent hMSCs into the cell cycle. Suggesting induction of p16 and $\mathrm{Rb}$ activation may be an important step in the process of irreversible senescence in hMSCs [27]. Furthermore, it has been shown low dose ionising radiation from rat bone marrow MSCs at 50 and $75 \mathrm{mGY}$ encouraged the proliferation of rat MSCs via MAPK/ERK signalling pathway activation [28].

When looking at the medium the cells are grown in, fibroblast growth factors are found to be a family of heparin binding polypeptide growth factors that are known to regulate cell proliferation and differentiation. In addition, the proliferation of human MSCs from bone marrow with FGF-2 culture medium supplementation was superior to population doubling-matched controls [29]. FGF-2 has been demonstrated in a dose dependant manner to stimulate the proliferation of mesenchyme derived progenitor cells cultures derived from adult bone. Although cells from older bones were less responsive to FGF-2 than those from younger bones, this is still a potential valuable factor in MSC culture [30]. Furthermore, the culturing of MSCs in human platelet lysate instead of fetal bovine serum resulted in a higher number of cell divisions [31].

\section{CONCLUSION}

The evidence on whether mesenchymal stem cell number in vivo is affected by donor age is inconclusive. Considering that older individuals have more fat in their bones, could studies have detected a difference by picking up on this artefact? The mesenchymal stem cell is an elusive cell. To this day we are unable to fully identify it, often we know within a group of cells from bone marrow there is a stem cell amongst the group. This surely presents challenges in quantification. The concept of replicative senescence poses wide ranging questions. Since stem cells should not really senesce are we highlighting these are not true stem cells? Considering in the past, research on mesenchymal stem cells bypassed legislation that was applicable to embryonic stem cells and this added to their attractiveness in research; whereas nowadays, they are subject to rigorous tests. Should we really be aiming for research with embryonic stem cells and avoiding the replicative senescence issue? By trying to combat replicative senescence are we in danger of leading to neoplastic transformations? This needs to be researched into and could have safety implications. Mesenchymal stem cells present an attractive option for treating an elderly generation from their own bone marrow, but many more studies are needed.

\section{ACKNOWLEDGEMENT}

With thanks to Professor Brendon Noble for having a discussion on some of the latest techniques in stem cell research. 


\section{CONFLICT OF INTEREST}

None declared.

\section{REFERENCES}

[1] Glowacki J. Influence of age on human marrow. Calcif Tissue Int 1995; 56(Suppl 1): S50-51.

[2] D'Ippolito G, Schiller PC, Ricordi C, Roos BA, Howard GA. Agerelated osteogenic potential of mesenchymal stromal stem cells from human vertebral bone marrow. J Bone Miner Res 1999; 14(7): 1115-22.

[3] de Girolamo L, Lopa S, Arrigoni E, Sartori MF, Baruffaldi Preis FW, Brini AT. Human adipose-derived stem cells isolated from young and elderly women: their differentiation potential and scaffold interaction during in vitro osteoblastic differentiation. Cytotherapy 2009; 11(6): 793-803.

[4] Roura S, Farré J, Soler-Botija C, Llach A, Hove-Madsen L, Cairó $\mathrm{JJ}$, et al. Effect of aging on the pluripotential capacity of human CD105+ mesenchymal stem cells. Eur J Heart Fail 2006; 8(6): 55563.

[5] Kronsteiner B, Wolbank S, Peterbauer A, et al. Human mesenchymal stem cells from adipose tissue and amnion influence T-cells depending on stimulation method and presence of other immune cells. Stem Cells Dev [Internet]. 2011 Mar 7 [cited 2011 Mar 26]; Available from: http: //www.ncbi.nlm.nih.gov/pubmed/ 21381973

[6] Chen Y, Yeh F, Yeh S, et al. MITR is a swith that promotes osteogenesis and inhibits adipogenesis of mesenchymal stem cells by inactivating PPAR \{gamma $\}$-2. J Biol Chem [Internet]. 2011 Jan 19 [cited 2011 Mar 26]; Available from: http: //www.ncbi.nlm. nih.gov/pubmed/21247904

[7] Viccica G, Francucci CM, Marcocci C. The role of PPAR $\gamma$ for the osteoblastic differentiation. J Endocrinol Invest 2010; 33(7 Suppl): 9-12.

[8] Katsara O, Mahaira LG, Iliopoulou EG, et al. Effects of Donor Age, Gender, and In Vitro Cellular Aging on the Phenotypic, Functional, and Molecular Characteristics of Mouse Bone MarrowDerived Mesenchymal Stem Cells. Stem Cells Dev 2011.

[9] Khan WS, Adesida AB, Tew SR, Andrew JG, Hardingham TE. The epitope characterisation and the osteogenic differentiation potential of human fat pad-derived stem cells is maintained with ageing in later life. Injury 2009; 40(2): 150-7.

[10] Zhou S, Greenberger JS, Epperly MW, et al. Age-Related Intrinsic Changes in Human Bone Marrow-Derived Mesenchymal Stem Cells and Their Differentiation to Osteoblasts. Aging Cell 2008; 7(3): 335-43.

[11] Sacchetti B, Funari A, Michienzi S, et al. Self-renewing osteoprogenitors in bone marrow sinusoids can organize a hematopoietic microenvironment. Cell 2007; 131(2): 324-36.

[12] Fan M, Chen W, Liu W, et al. The effect of age on the efficacy of human mesenchymal stem cell transplantation after a myocardial infarction. Rejuvenation Res 2010; 13(4): 429-38.

[13] Shibata KR, Aoyama T, Shima Y, et al. Expression of the p16INK4A gene is associated closely with senescence of human mesenchymal stem cells and is potentially silenced by DNA methylation during in vitro expansion. Stem Cells 2007; 25(9): 2371-82.

[14] Stolzing A, Jones E, McGonagle D, Scutt A. Age-related changes in human bone marrow-derived mesenchymal stem cells: consequences for cell therapies. Mech Ageing Dev 2008; 129(3): 163-73.

[15] Hayflick L, Moorhead PS. The serial cultivation of human diploid cell strains. Exp. Cell Res 1961; 25: 585-621.

[16] Werner C, Gensch C, Pöss J, Haendeler J, Böhm M, Laufs U. Pioglitazone activates aortic telomerase and prevents stress-induced endothelial apoptosis. Atherosclerosis 2011.

[17] Ghildiyal M, Zamore PD. Small silencing RNAs: an expanding universe. Nat Rev Genet 2009; 10(2): 94-108.

[18] Grillari J, Hackl M, Grillari-Voglauer R. miR-17-92 cluster: ups and downs in cancer and aging. Biogerontology 2010; 11(4): 501506.

[19] Li SKM, Smith DK, Leung WY, et al. FoxM1c counteracts oxidative stress-induced senescence and stimulates Bmi-1 expression. J Biol Chem 2008; 283(24): 16545-53.

[20] Wagner W, Horn P, Castoldi M, et al. Replicative senescence of mesenchymal stem cells: a continuous and organized process. PLoS ONE 2008; 3(5): e2213.

[21] Zhang J, Lian Q, Zhu G, et al. A human iPSC model of Hutchinson Gilford Progeria reveals vascular smooth muscle and mesenchymal stem cell defects. Cell Stem Cell 2011; 8(1): 31-45.

[22] Kongsuwan K, Webb E, Housiaux P, Adams JM. Expression of multiple homeobox genes within diverse mammalian haemopoietic lineages. EMBO J 1988; 7(7): 2131-8.

[23] Fehrer C, Brunauer R, Laschober $\mathrm{G}$ et al. Reduced oxygen tension attenuates differentiation capacity of human mesenchymal stem cells and prolongs their lifespan. Aging Cell 2007; 6(6): 745-57

[24] Laschober GT, Brunauer R, Jamnig A, Fehrer C, Greiderer B, Lepperdinger G. Leptin receptor/CD295 is upregulated on primary human mesenchymal stem cells of advancing biological age and distinctly marks the subpopulation of dying cells. Exp Gerontol 2009; 44(1-2): 57-62.

[25] Colter DC, Class R, DiGirolamo CM, Prockop DJ. Rapid expansion of recycling stem cells in cultures of plastic-adherent cells from human bone marrow. Proc Natl Acad Sci USA 2000; 97(7): 3213-8.

[26] Simonsen JL, Rosada C, Serakinci N, et al. Telomerase expression extends the proliferative life-span and maintains the osteogenic potential of human bone marrow stromal cells. Nat Biotechnol 2002; 20(6): 592-6.

[27] Lee JS, Lee MO, Moon BH, Shim SH, Fornace AJ Jr, Cha HJ. Senescent growth arrest in mesenchymal stem cells is bypassed by Wip1-mediated downregulation of intrinsic stress signaling pathways. Stem Cells 2009; 27(8): 1963-75.

[28] The Low-dose Ionizing Radiation Stimulates Cell Proliferation via Activation of the MAPK/ERK Pathway in Rat Cultured Mesenchymal Stem Cells. J Radiat Res 2011.

[29] Auletta JJ, Zale EA, Welter JF, Solchaga LA. Fibroblast Growth Factor-2 Enhances Expansion of Human Bone Marrow-Derived Mesenchymal Stromal Cells without Diminishing Their Immunosuppressive Potential Stem Cells Int 2011; 2011: 2351-76.

[30] Ou G, Charles L, Matton S, Rodner C, Hurley M, Kuhn L, Gronowicz G. Fibroblast growth factor-2 stimulates the proliferation of mesenchyme-derived progenitor cells from aging mouse and human bone. J Gerontol A Biol Sci Med Sci 2010; 65(10): 1051-9.

[31] Colter DC, Class R, DiGirolamo CM, Prockop DJ. Rapid expansion of recycling stem cells in cultures of plastic-adherent cells from human bone marrow. Proc Natl Acad Sci USA 2000; 97(7): 3213-8. 